\title{
Heterodyne speckle velocimetry
}

\author{
M. D. Alaimo \\ Dipartimento di Fisica, Università di Milano and CNR-INFM, Via Celoria 16, Milano I-20133, Italy \\ D. Magatti and F. Ferri \\ Dipartimento di Fisica e Matematica, Università dell'Insubria and CNR-INFM, Via Valleggio 11, \\ Como I-22100, Italy
}

\author{
M. A. C. Potenza ${ }^{\text {a) }}$ \\ Dipartimento di Fisica, Università di Milano and CNR-INFM, Via Celoria 16, Milano I-20133, Italy
}

(Received 27 June 2005; accepted 5 April 2006; published online 8 May 2006)

\begin{abstract}
We present a simple method for fluid velocimetry based on the velocity of the heterodyne speckles generated by tracking particles illuminated with coherent light. It works in real time and provides instantaneous two-dimensional velocity mappings in the direction orthogonal to the optical axis, independently of the particle concentration and size, also for subwavelength particles. It also provides the velocity distribution of the fluid over the entire sample thickness. The method has been quantitatively tested by using the motions of rigid diffusers and applied for mapping the flow of a confined fluid. (C) 2006 American Institute of Physics. [DOI: 10.1063/1.2200396]
\end{abstract}

Modern experimental fluid dynamics relies on the instantaneous measurements of the velocity fields of a moving fluid seeded with tracking particles. One of the most popular techniques devoted to this aim is the particle imaging velocimetry (PIV), which can be used under different modes of operation. $^{1-3}$ In its simplest realization, the tracers are illuminated by a thin sheet of light and imaged in the perpendicular direction. By measuring the tracer displacement at two closely spaced times, one recovers the two-dimensional (2D) in-plane velocity of the fluid. Since the spatial resolution is $\Delta r \sim \lambda /(2 \mathrm{NA})$ where NA is the numerical aperture of the imaging system, and the depth of focus is $\Delta z \sim \lambda /(2 \mathrm{NA})^{2}$, a high resolution can be achieved only with a small depth of focus. Thus, the effective volume of the probed fluid is very thin. Depending on the NA, the tracer concentration has to be low enough, otherwise their positions cannot be resolved. In the latter case, the image becomes speckled but the technique still works properly and is named laser speckle velocimetry (LSV). ${ }^{4}$

Many difficulties can be overcome with the use of the holographic PIV (HPIV), ${ }^{5,6}$ which recovers the threedimensional (3D) tracer positions with a diffraction limited resolution. It almost does not impose any constraint on the thickness of the sample, thus appearing to be the most promising method for a full 3D characterization of a fluid flow. However, HPIV presents several drawbacks: the setup may be rather complex, the data reduction is usually not straightforward (and carried out not in real time), and the scattering signal of each single particle has to be strong enough to beat the system noise. This is related to the system sensitivity and determines the minimum size of the tracers, which are typically of the order of microns, or larger. ${ }^{6}$

In this letter we propose and discuss a very simple and fast method for 2D fluid velocimetry, that provides instantaneous velocity mappings and velocity distributions of a fluid moving in a plane. It profits from the contributions coming from the entire volume thickness, works with tracer sizes well below the resolution of the collection optic, and rela-

\footnotetext{
${ }^{a)}$ Electronic mail: marco.potenza@unimi.it
}

tively high concentrations can be used as well. Finally, the data reduction scheme is fast enough, allowing for an easy real-time analysis. The method is related to the technique called heterodyne near field scattering (HNFS). ${ }^{7,8}$ It requires a remarkably simple optical setup (see Fig. 1) similar to the in-line HPIV, in which a large collimated laser beam of diameter $D$ and wavelength $\lambda$ is sent onto a square cell containing a fluid seeded with small tracking particles of average diameter $d$. By using an imaging system, a charge coupled device (CCD) detects the intensity of light falling onto a plane located at a close distance $z$ from the sample. Here, the intensity distribution is the result of the interference between the strong transmitted beam and the weak light scattered by the tracers. If $\theta_{\max }$ denotes the maximum scattering angle, for small particles $(d \leqslant \lambda) \theta_{\max }$ is set by the NA of the collection optic $\left(\theta_{\max } \sim \mathrm{NA}\right)$, while for very large particles it is ultimately determined by the maximum angle the particle can scatter at $\left(\theta_{\max } \sim \lambda / 2 d\right)$. In any case, the sample region which contributes to the signal is $D^{*} \sim 2 z \theta_{\max }$, and the near field condition can be written as $D^{*} \ll D$.

If the scattered field $e_{s}$ is negligible with respect to the transmitted field $e_{0}\left(e_{S} \ll e_{0}\right)$, the intensity distribution at the time $t$ and position $\mathbf{r}$ on the observation plane is

$$
f(\mathbf{r}, t)=\left|e_{0}\right|^{2}+2 \operatorname{Re}\left\{e_{0}^{*} e_{S}(\mathbf{r}, t)\right\},
$$

where $\left|e_{0}\right|^{2}$ is the static and almost uniform transmitted intensity, while the last term is a stochastic zero-average fluc-

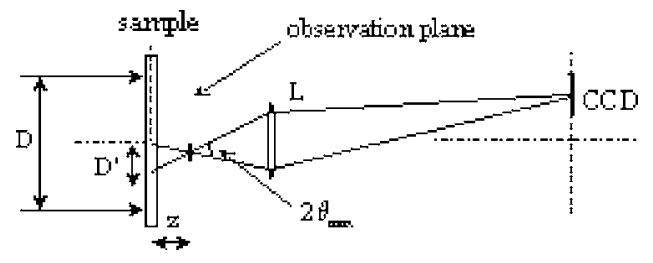

FIG. 1. Schematic optical layout. A collimated laser beam of diameter $D$ illuminates a thin cell containing the fluid seeded with tracking particles. The interference between the transmitted and the scattered light is recorded at a close distance $z$ from the sample through a magnifying optic. The collected light comes from a region of the sample with diameter $D^{*} \ll D$. 
tuating term which is called heterodyne signal. Note that with $e_{0}$ being almost uniform, the signal $2 \operatorname{Re}\left\{e_{0}^{*} e_{S}(\mathbf{r}, t)\right\}$ is a direct measurement of the scattered electric field.

If the sample is made of $N$ particles, we can write the scattered field $e_{S}(\mathbf{r}, t)$ as

$$
e_{S}(\mathbf{r}, t)=e_{0} \sum_{i=1}^{N^{*}(\mathbf{r}, t)} a_{i}(\mathbf{r}, t) e^{-j \phi_{i}(\mathbf{r}, t)},
$$

in which $a_{i}$ and $\phi_{i}$ are the amplitude and the phase of the field scattered by the $i$ th particle. The sum is extended only to the $N^{*}<N$ particles lying within the region $D^{*}$ centered on the back projection of $\mathbf{r}$ over the sample. For randomly distributed particles, the sum behaves as a $2 \mathrm{D}$ random walk $\left(N^{*} \gg 1\right)$ and therefore, for any time $t, e_{S}$ exhibits a speckled appearance, with speckles of $\operatorname{size}^{9} \delta \sim \lambda / 2 \theta_{\max }$. Note that, when $\theta_{\max } \sim \lambda / 2 d$, the speckles are determined only by the size of the particles ${ }^{10}(\delta \sim d)$, while when $\theta_{\max } \sim \mathrm{NA}$, they depend on the collection optic (subjective speckles ${ }^{11}$ ).

The classical way to characterize the spatio-temporal statistical properties of $e_{S}(\mathbf{r}, t)$ is to compute its mutual coherence function defined as ${ }^{12}$

$$
G(\mathbf{x}, \tau)=\left\langle e_{S}(\mathbf{r}, t) e_{S}^{*}(\mathbf{r}+\mathbf{x}, t+\tau)\right\rangle,
$$

in which $\langle\cdots\rangle$ denotes ensemble average. By using Eq. (2) and noting that all the terms with $i \neq j$ vanish, we obtain

$$
G(\mathbf{x}, \tau)=\left|e_{0}\right|^{2} \sum_{i=1}\left\langle a_{i}(\mathbf{r}, t) a_{i}^{*}(\mathbf{r}+\mathbf{x}, t+\tau) e^{-j \Delta \phi_{i}(\mathbf{r}, \mathbf{x}, t, \tau)}\right\rangle,
$$

where $\Delta \phi_{i}(\mathbf{r}, \mathbf{x}, t, \tau)=\phi_{i}(\mathbf{r}+\mathbf{x}, t+\tau)-\phi_{i}(\mathbf{r}, t)$ and the sum is extended to all those particles which give contribution at time $t$ in $\mathbf{r}$ and at time $t+\tau$ in $\mathbf{r}+\mathbf{x}$. Here $G(\mathbf{x}, 0)$ represents the spatial autocorrelation function (ACF) of the scattered field, while $G(0, \tau)$ is the temporal ACF carrying information about particle motions.

Let us now suppose, for the sake of simplicity, that all the particles move with the same velocity $\mathbf{v}$ transverse to the optical axis. Thus, the scattered field at the observation plane in $\mathbf{r}+\Delta \mathbf{r}$ at the time $t+\tau$ is simply a "shifted version" of the field in $\mathbf{r}$ at the time $t$, and

$$
\phi_{i}(\mathbf{r}+\Delta \mathbf{r}, t+\tau)=\phi_{i}(\mathbf{r}, t),
$$

in which $\Delta \mathbf{r}=\mathbf{v} t$. By using Eq. (5) we obtain

$$
G(\mathbf{x}+\Delta \mathbf{r}, \tau)=G(\mathbf{x}, 0),
$$

showing that, under a rigid displacement of all the scatterers orthogonally to the optical axis, the cross-correlation function $(\mathrm{CCF}) G(\mathbf{x}, \tau)$ is simply a shifted version of the ACF
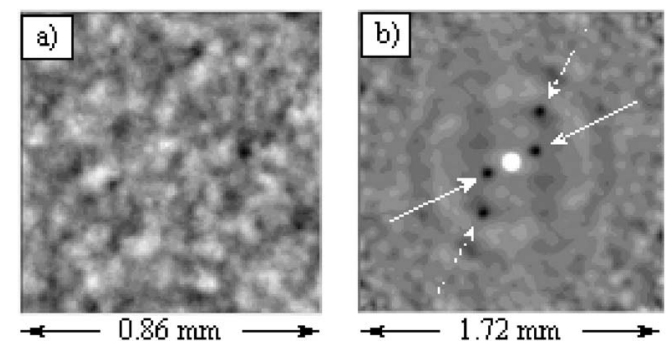

FIG. 2. (a) Differential heterodyne signal $\delta f(\mathbf{r}, t, \tau)$ obtained from two semitransparent diffusers moving with velocities $\mathbf{v}_{1}$ and $\mathbf{v}_{2}$ transversally to the optical axis; the lag time was $\tau=0.1 \mathrm{~s}$. (b) Spatial autocorrelation function of $\delta f(\mathbf{r}, t, \tau)$ obtained by averaging 100 frames. The dark spots correspond to

$\mathbf{v}_{1}=3.29 \mathrm{~mm} / \mathrm{s}$ (dashed arrow) and $\mathbf{v}_{2}=1.47 \mathrm{~mm} / \mathrm{s}$ (solid arrow). tained for $\tau=0.1 \mathrm{~s}[\mathrm{Fig}$. 2(a)] is a uniform speckle field
Downloaded 31 May 2006 to 193.206 .163 .233 . Redistribution subject to AlP license or copyright, see http://apl.aip.org/apl/copyright.jsp
$G(\mathbf{x}, 0)$. Two close displacements $\Delta \mathbf{r}$ apart can be resolved only if $\Delta \mathbf{r} \geqslant \delta, \delta$ being the width of $G(\mathbf{x}, 0)$ (average speckle size). Equation (6) holds when the longitudinal displacements $\Delta z$ during the time $\tau$ are negligible with respect to the axial speckle length, given by ${ }^{13} \delta_{z} \sim 2 \lambda /\left(2 \theta_{\max }\right)^{2}$. Otherwise, the CCF $G(\mathbf{x}, \tau)$ changes, and its integral becomes smaller than the one associated with ACFs.

We now discuss the key property of the method, namely, the linearity of the function $G(\mathbf{x}, \tau)$. Equation (4) shows that $G(\mathbf{x}, \tau)$ is a weighted sum of contributions from all those particles that have been displaced by $\mathbf{x}$ during the time $\tau$, the weight factor being associated with particle size via the amplitude $a_{i}$. Notice that, since the method works by taking two snapshots at a time distance $\tau$ (exactly as in PIV), it can only associate with each displacement $\mathbf{x}$ an average velocity $\langle\mathbf{v}\rangle$ $=\mathbf{x} / \tau$. Moreover, since different particles can undergo different displacements $\mathbf{x}$, the assumption of equal velocities made for working out Eq. (5) can be dropped. Thus, provided that the particles are uniformly distributed and dragged within the fluid (as commonly used in velocimetry), the amplitude of $G(\mathbf{x}, \tau)$ is proportional to the fraction of fluid that has moved by $\langle\mathbf{v}\rangle=\mathbf{x} / \tau$, regardless of particle size polydispersity.

Another important feature of the technique is its ability to provide 2D mappings of the fluid velocities. This property derives from the almost local character of Eq. (4): the displacement of the speckles at a given position $\mathbf{r}$ of the sensor is determined only by the motion of the $N^{*}$ scatterers inside the portion $D^{*}$ of the sample (see Fig. 1). Thus, if we locate the observation plane very close to or inside the sample, we establish a one-to-one mapping between the position on the sensor and a small section of the cell. In this way a $2 \mathrm{D}$ mapping of the fluid motion in the plane transverse to the optical axis is possible, allowing the study of transversal velocity gradients present in the fluid.

The data analysis is carried out by adopting the double frame analysis suggested in Ref. 8, in which the frames are acquired at temporal distance $\tau$. The difference between $f(\mathbf{r}, t)$ and $f(\mathbf{r}, t+\tau)$ is used to define the differential heterodyne signal

$$
\delta f(\mathbf{r}, t, \tau)=2 \operatorname{Re}\left\{e_{0}^{*}\left[e_{s}(\mathbf{r}, t+\tau)-e_{s}(\mathbf{r}, t)\right]\right\},
$$

which is independent of $\left|e_{0}\right|^{2}$ and then of any static stray light contribution. The autocorrelation function of $\delta f(\mathbf{r}, t, \tau)$ is

$$
g_{\tau}(\mathbf{x})=4\left|e_{0}\right|^{2} \operatorname{Re}\{2 G(\mathbf{x}, 0)-G(\mathbf{x}, \tau)-G(-\mathbf{x}, \tau)\} .
$$

The first term is the ACF of the speckle field, is positive, and brings no information about the motion; conversely, the last two are CCF terms, are negative, and depend on particle motion. Note that $g_{\tau}(\mathbf{x})$ is symmetrical with respect to $\mathbf{x}=0$, and carries information only about the direction and modulus of the fluid velocity, but not about its versus.

A quantitative test of the method was carried out by using two diffusers moving in different directions perpendicularly to the optical axis, at velocities $\mathbf{v}_{1}=3.29 \mathrm{~mm} / \mathrm{s}$ and $\mathbf{v}_{2}=1.47 \mathrm{~mm} / \mathrm{s}$. In order to obtain a strong transmitted beam, each diffuser was made by sticking together a ground and a transparent glass with an optical oil in between. The two diffusers were illuminated with a $\mathrm{He}-\mathrm{Ne}$ laser beam $\left(\lambda=0.6328 \mu \mathrm{m}, D=20 \mathrm{~mm}\right.$ at $\left.1 / e^{2}\right)$, the collection optic was a $4 \times$ microscope objective $(\mathrm{NA}=0.1)$, and the observation plane was at a distance $z=70 \mathrm{~mm}$. The signal $\delta f(\mathbf{r}, t, \tau)$ obtained for $\tau=0.1 \mathrm{~s}$ [Fig. 2(a)] is a uniform speckle field 

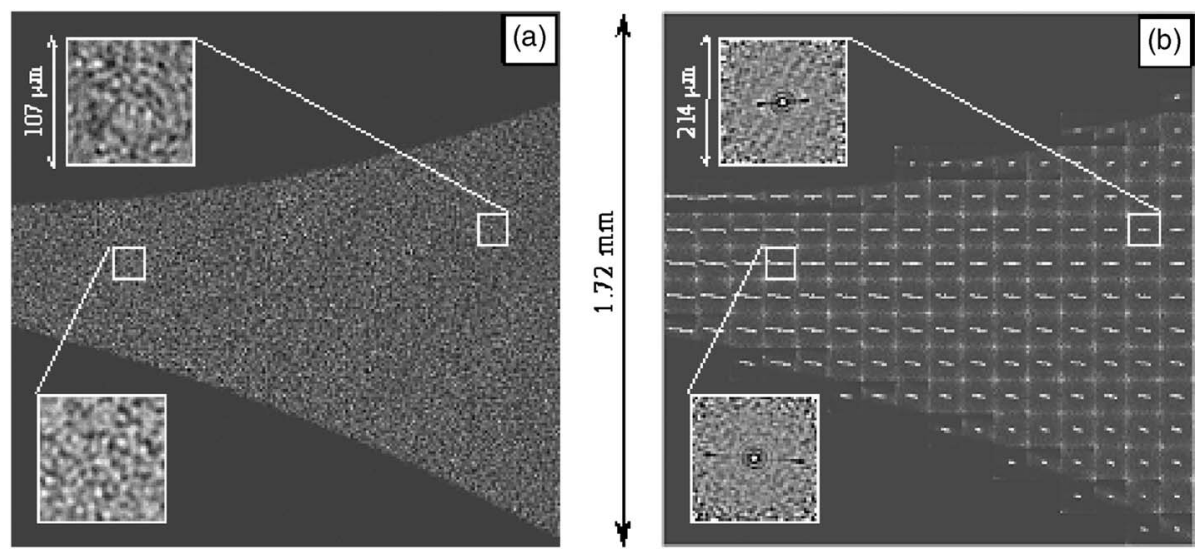

FIG. 3. (a) Differential heterodyne signal $\delta f(\mathbf{r}, t, \tau)$ for $\tau=0.1 \mathrm{~s}$ from $300 \mathrm{~nm}$ latex particle flowing from right to left inside a funnel shaped cell. (b) Mapping of the correlation functions $g_{\tau}(\mathbf{x})$ obtained by dividing Fig. 3 (a) into $16 \times 16$ subframes. The line segments are guide to the eye, connecting the two darkest spots present in each subframe. The velocities corresponding to the darkest spots of the upper and lower insets are 439 and $787 \mu \mathrm{m} / \mathrm{s}$, respectively.

where the displacement is completely hidden. Conversely, the correlation $g_{\tau}(\mathbf{x})$ [Fig. 2(b)] does carry this information in the double twin dark (negative) spots corresponding to the CCF $G(\mathbf{x}, \tau)$. The central white (positive) spot is associated with the ACF $G(\mathbf{x}, 0)$. The values for the two velocities were correct within the accuracy of the system, which is set by the (effective) pixel size and lag time $\tau(\sim 17 \mu \mathrm{m} / \mathrm{s})$. The linearity of the system was by comparing the amplitudes of the various correlation peaks.

The method was also tested on a real fluid sample made of a water suspension of latex particles, $300 \mathrm{~nm}$ in diameter, fluxed at $\sim 10^{-3} \mathrm{ml} / \mathrm{s}$ inside a square $1.5 \mathrm{~mm}$ thick cell whose width was tapered as a funnel. The setup was the same as above, but with $z=0$. The signal $\delta f(\mathbf{r}, t, \tau)$ for $\tau=0.1 \mathrm{~s}$ [Fig. 3(a)] shows a quite uniform speckle field inside the entire funnel, with speckles of size $\delta \sim 3 \mu \mathrm{m}$ $(\delta \sim \lambda / 2 \mathrm{NA})$. The analysis, carried out on an array of $16 \times 16$ subframes $\left(\sim 107 \times 107 \mu \mathrm{m}^{2}\right)$, is shown in Fig. 3(b), where the segments indicate the direction and modulus of the various velocities. As expected for an uncompressible viscous fluid undergoing a laminar flux, the velocity increases both in the middle of the channel and as the funnel neck gets narrower. A simple quantitative check showed that the fluid flux is conserved. Note that, similarly to what happens in PIV, the natural limit to the smallness of the tracers is determined by their Brownian motions. ${ }^{14}$ However, in our case (300 nm particles $\tau=0.1 \mathrm{~s}$ the r.m.s. displacement is $\sqrt{\left\langle s^{2}\right\rangle}$ $\sim 1.2 \mu \mathrm{m} \quad\left(\left\langle\mathrm{s}^{2}\right\rangle=2 D \tau, \quad D=7.3 \times 10^{-12} \mathrm{~m}^{2} / \mathrm{s} \quad\right.$ in water at $300 \mathrm{~K})$. Since $\sqrt{\left\langle s^{2}\right\rangle}$ is smaller than the speckle size $\delta$ $\sim 3 \mu \mathrm{m}$, diffusion does not play an important role.

The analysis of each subframe [insets of Fig. 3(b)] showed that the CCF terms look like elongated streams This is a clear indication of the presence of a velocity distribution inside that region, accordingly to the Poiseuille condition.

In conclusion we have proposed a simple and sensitive method for 2D real-time velocimetry in fluids seeded with small particles. We suggest to call this method heterodyne speckle velocimetry (HSV), because it measures the velocity of the heterodyne speckles originated from the interference between the strong transmitted beam and the weak scattered light. The heterodyne condition is a key feature of the method because it allows to measure the sample field-field CCF $G(\mathbf{x}, \tau)$, which is linear in the number of scattering particles displaced by a distance $\mathbf{x}$ over the time $\tau$. Thus, although no information is obtained about the velocity as a function of the longitudinal coordinate (optical axis $z$ ), the velocity distribution over the entire sample thickness can be recovered. This is the main difference of HSV with respect to the classical LSV technique, which is not endowed with such linearity properties. Moreover, HSV is more sensitive than LSV because it collects the forward scattered light, which is stronger than the light scattered in any other direction. HSV adopts a very simple and compact optical layout similar to in-line HPIV, but since the analysis relies on speckle pattern, the tracer size can be very small (submicron) and their concentration very high. This is a great advantage with respect to any imaging technique (PIV or HPIV) which, relying on the light scattered by single particles, becomes difficult for very small tracers. The optical layout of HSV is very robust and almost free from any alignment procedure, all features of the most importance in many practical applications. In particular, the subtraction of the stray light allows HSV to work in difficult conditions where optical surfaces are affected by dust or droplets or any other disturbance.

Finally, we would like to mention the main limitation of the method. It is related to the hypothesis of pure transversal motion. Works are in progress to overcome this limitation.

The authors thank M. Giglio for early suggestions regarding the ideas which have brought to the development of the technique proposed in this work. The authors also thank D. Brogioli for useful discussions. Financial support from INFM (project PAIS-NFS) is kindly acknowledged.

${ }^{1}$ R. J. Adrian, Annu. Rev. Fluid Mech. 23, 261 (1991).

${ }^{2}$ M. Raffel, C. Willert, and J. Kompenhans, Particle Image Velocimetry, a Practical Guide (Springer, Berlin, 1998).

${ }^{3}$ Proceedings of the EUROPIV Workkshop, Zaragoza, Spain, 31 March-1 April 2003, edited by M. Stanislas, J. Westerweel, and J. Kompenhans (Springer, New York, 2004).

${ }^{4}$ T. D. Dudderar, R. Meynart, and P. G. Simpkins, OLE 9, 163 (1988).

${ }^{5}$ D. H. Barnhart, R. J. Adrian, and G. C. Papen, Appl. Opt. 33, 7159 (1994).

${ }^{6}$ J. O. Sherer and L. P. Bernal, Appl. Opt. 36, 9309 (1997).

${ }^{7}$ D. Brogioli, A. Vailati, and M. Giglio, Appl. Phys. Lett. 81, 4109 (2002).

${ }^{8}$ F. Ferri, D. Magatti, D. Pescini, M. A. C. Potenza, and M. Giglio, Phys. Rev. E 70, 041405 (2004).

${ }^{9}$ J. W. Goodman, in Laser Speckle and Related Phenomena, edited by J. C. Dainty (Springer, Berlin, 1975).

${ }^{10}$ M. Giglio, M. Carpineti, and A. Vailati, Phys. Rev. Lett. 85, 1416 (2000).

${ }^{11}$ A. E. Ennos, in Laser Speckle and Related Phenomena, edited by J. C. Dainty (Springer, Berlin, 1975).

${ }^{12}$ J. W. Goodman, Statistical Optics (Wiley, New York, 1985), p. 174.

${ }^{13}$ G. P. Weigelt in Speckle Metrology, edited by R. K. Erf (Academic, New York, 1978), p. 168.

${ }^{14}$ J. G. Santiago, S. T. Wereley, C. D. Meinhart, D. J. Beebe, and R. J. Adrian, Exp. Fluids 25, 316 (1998). 